\title{
Eficacia de la terapia cognitivo conductual en la prevención de la depresión posparto
}

\author{
Bernardo Nardi ${ }^{1}$, Cesario Bellantuono ${ }^{1}$, Andrés Moltedo-Perfetti. $2 a$ \\ ${ }^{1}$ Università Politecnica delle Marche, Italia. ${ }^{2}$ Pontificia Universidad Católica de Valparaíso, Chile. \\ a Psicólogo.
}

\section{RESUMEN}

Objetivo: Evaluar la eficacia de la terapia cognitivo conductual (TCC) en la prevención de la depresión posparto (DPP) en mujeres embarazadas consideradas en riesgo de padecerla. Método: Revisión bibliográfica en PubMed, Medline, PsychInfo, Embase y de la Cochrane Library de publicaciones efectuadas entre enero de 1991 y junio de 2012 respecto de investigaciones sobre la eficacia de la TCC en la prevención de la DPP. Resultados: De la literatura analizada se concluye que la depresión en el embarazo requiere de un manejo eficiente tanto para tratar los síntomas de la depresión preparto (DAP), como de prevenir la DPP. Mientras diversos estudios han demostrado la eficacia de la TCC en el tratamiento de la DPP, sólo pocos estudios controlados se han centrado sobre su eficacia en la prevención de la DPP. La eficacia en la prevención de la DPP en mujeres en riesgo durante el embarazo ha sido apoyada sólo por pocos estudios, los cuales presentan algunas limitaciones metodológicas. Conclusión: Resultan necesarios estudios clínicos para confirmar la eficacia de tal estrategia de prevención psicoterapéutica en las mujeres en riesgo de DPP.

\section{PALABRAS CLAVE: Embarazo, depresión posparto, terapia cognitivo-conductual, modelo cognitivo post-racionalista}

\section{SUMMARY}

Aims: To evaluate the efficacy of cognitive-behavioral therapy (CBT) in the prevention of postnatal depression (PD) in pregnant women at risk. Methods: PubMed, Medline, PsychInfo, Embase, and the Cochrane Library data bases were searched from January 1991 to June 2011 to review studies on the efficacy of CBT in the prevention of PD. Results: The literature analyzed recommends that depression in pregnancy requires an efficient management to provide mother's symptoms relief as well as to prevent PD. While several studies demonstrated the efficacy of CBT in the treatment of PD, only a few controlled studies focused on its efficacy in the prevention of PD in women identified at risk during pregnancy. The efficacy of CBT in preventing PD in pregnant women at risk is supported by only a few studies, presenting some methodological flaws. Conclusion: Better designed trials are needed to strongly support the efficacy of such psychotherapeutic preventive strategy in women at risk for PD.

KEY WORDS: Pregnancy, postnatal depression, cognitive-behavioral therapy, post rationalist cognitive approach 


\section{INTRODUCCIÓN}

Depresión y embarazo. A pesar de que el embarazo es considerado como un momento de bienestar emotivo para una mujer, muchos estudios demuestran que un número relevante de mujeres embarazadas presentan síntomas depresivos, estimándose que está presente entre el $7-11 \%$ de las mujeres durante el primer trimestre y del $8-13 \%$ durante el segundo y tercer trimestre del embarazo (1-4).

La depresión durante el embarazo (o depresión preparto DAP) es, muchas veces, subvalorada y no diagnosticada $(5,6)$. Diversos estudios han señalado la existencia de un continuum existente entre la depresión durante el embarazo y la depresión en el período posnatal (7). Así, en los últimos 25 años muchos artículos se han focalizado en la depresión posparto (DPP), sin otorgarle a la depresión en el embarazo una importancia significativa (8-11).

Con el término de DPP se entiende un episodio de tipo depresivo que se manifiesta en el período posparto (12) que se articula en una serie de síntomas determinando una grave limitación respecto del funcionamiento global y la calidad de vida de la mujer, con consecuencias también importantes sobre las dinámicas relacionales con su pareja, así como con su hijo y, potencialmente, en el desarrollo psíquico de este último.

De acuerdo con Gjerdingen (13) la DPP representa la complicación más considerable del puerperio, con una incidencia entre el $12-16 \%$, sin embargo estas cifras pueden variar de acuerdo a los estudios entre un $10-20 \%$ según el método y el momento de diagnóstico $(14,15)$, pero siempre considerando que los casos más leves pueden no ser reconocidos ni diagnosticados (16).

La prevalencia de la DPP oscila entre el $8-30 \%$, observándose cifras tres veces más alta en los países emergentes que en los países desarrollados (17-20). En Chile, los estudios han informado una incidencia de $9,2 \%$ y una prevalencia de $10,2 \%$ (21), o una incidencia de $8,8 \%$ y una prevalencia de $20,5 \%$ (22).

Como se ha señalado, se trata de un desorden depresivo mayor que se manifiesta en el período posparto (12), es decir, los síntomas de la depresión en el embarazo y en el posparto no difieren de aquellos de la depresión en otros momentos del ciclo vital (23).

Desde un punto de vista diagnóstico, se evidencia un compromiso general de la capacidad funcional de un cuadro sintomatológico que dura por al menos una semana y se caracteriza por manifestaciones tales como baja de ánimo, fatiga, trastorno psicomotor, trastornos del sueño y del apetito, pre- sencia de sentimientos de culpa y, tal vez, ideación suicida (24), pero que en la DPP se suele apreciar una tendencia al llanto y labilidad emotiva (23).

Esta sintomatología habitualmente no se diagnostica, porque en muchos casos suele asumirse que se trata de cambios emocionales normales y no de depresiones, especialmente cuando su aparición es tardía respecto del parto, o porque los profesionales se centran más en la salud física que en la mental de la madre (23).

La DPP debe de ser diferenciada de la disforia posparto ("maternity blues") y la psicosis puerperal. La disforia posparto consiste en un trastorno transitorio de la emotividad muy frecuente en la primera semana del posparto que se puede extender hasta por 10-15 días (25-28) y que tiene una incidencia entre $50-84 \%(25,29)$, manifestándose mediante síntomas como llanto, irritabilidad, humor deprimido y ansioso (26). Por lo general no requiere tratamiento específico que vaya más allá de la comprensión, escucha y empatía (28).

Por su parte, la psicosis puerperal es un trastorno psiquiátrico que incide entre el $0,1-0,2 \%$ de la población (30) caracterizado por delirios, alucinaciones y una notable reducción de la función psíquica de la mujer (31). Dentro de los desórdenes posnatales es seguramente el más grave en términos de riesgos potenciales para la salud de la madre y del niño (32-34).

Como se ha señalado, si la DAP no es adecuadamente tratada puede producir daño significativo tanto para la madre como para el feto (35), aumentando el riesgo de DPP (36). Por este motivo resulta esencial que las intervenciones terapéuticas sean adecuadas (37-40).

Dada la multifactoriedad del fenómeno, resulta claro que no es posible pensar en una única modalidad de tratamiento como terapia eficaz para todas las situaciones, ante una patología que combina la participación de factores biológicos y psicológicos (41). Dentro de las diferentes opciones de intervención se incluye el tratamiento farmacológico que comprende la prescripción de fármacos antidepresivos (AD), terapia de grupo, consejería y distintos modelos de psicoterapia $(23,42-47)$.

Es una experiencia común que muchas mujeres que padecen de DAP no quieran utilizar fármacos a causa del posible riesgo de malformaciones mayores, de complicaciones perinatales, así como de potenciales efectos negativos sobre el desarrollo del niño en el corto o largo plazo $(48,49)$. Así, la Guía Clínica para el tratamiento de personas con depresión (23) señala como contraindicados durante el primer trimestre del embarazo a la paroxetina, las benzodiacepinas, el litio, la carbamazepina y el 
ácido valproico.

De manera similar, en lo que respecta al tratamiento de la DPP, no obstante que numerosos estudios han demostrado la eficacia y la seguridad de los tratamientos farmacológicos (50-52), muchas madres se muestran titubeantes al tomar $A D$, por temor respecto de los efectos negativos potenciales ligados a la transmisión del fármaco al niño a través de la leche materna (53).

La American Academy of Pediatrics ha clasificado la mayor parte de los $A D$ (con la excepción de la sertralina y paroxetina) como fármacos no compatibles con el amamantamiento (54). Por lo mismo, resulta importante establecer la real eficacia de las intervenciones no farmacológicas tanto en el manejo de la DAP como en la prevención de la DPP $(44,55)$, ya que los modelos psicoterapéuticos son recomendados como primera opción en el caso de depresión leve y moderada $(56,57)$. Específicamente, en Chile, la Guía Clínica para el tratamiento de personas con depresión (23) señala que para el tratamiento de la depresión durante el embarazo o posparto, hay que iniciar el tratamiento con opciones terapéuticas no farmacológicas, específicamente intervenciones ambientales y psicoterapia.

En dicho rango de depresión, como lo han documentado estudios controlados, la terapia cognitivo-conductual (TCC) resulta tener una eficacia par respecto de la mayor parte de los $A D(58,59)$.

Terapia cognitivo-conductual y embarazo. Como se sabe, la TCC comprende tres aproximaciones clínicas principales:

1. Terapia conductual: desarrollada principalmente aplicando a la psicología la metodología científica y la aproximación inductiva e hipotética deductiva de la medicina experimental. Según este enfoque, un síntoma representa una respuesta errada aprendida durante un proceso aprendizaje. Todas las intervenciones clínicas, por diversas que sean, buscan cambiar la relación entre estímulos para generar un nuevo aprendizaje y así cambiar la respuesta (el comportamiento patológico o no funcional).

2. Enfoques cognitivos racionalistas: el comportamiento es correlacionado con la manera con la cual el sujeto se concibe a sí mismo y al mundo. Específicamente, la realidad es vista como objetivamente cognoscible y, en consecuencia, modificable de acuerdo a parámetros válidos para todos. Los trastornos psicocomportamentales son entendidos como consecuencia de disfunciones cognitivas, que se buscan reducir, controlar y, si es posible, eliminar a través de intervenciones tendientes a afrontar (coping) y a manejar de manera más ade- cuada las situaciones estresantes, tanto a través de terapias reforzadoras de la habilidad de manejar o gestionar, como de resolución de los problemas.

3. Enfoque cognitivo post-racionalista: para esta evolución constructivista del modelo anterior, no es posible tener una idea objetivamente unívoca de la realidad, ya que ésta es conocible sólo a través de la subjetividad del individuo que la explora y vive. Ella es así construida por el sujeto mediante las herramientas emocionales y cognitivas de las que dispone en cierto momento vital y de acuerdo a sus propias referencias. La psicoterapia, desde esta concepción, utiliza las reacciones emotivas, que emergen de las perturbaciones estratégicamente orientadas, generadas por el terapeuta, a través de la "moviola", de episodios significativos en los que se efectúan reformulaciones que evidencien al sujeto su manera personal de funcionar y de autoreferirse su experiencia inmediata. Así, el paciente puede descubrir otra modalidad emotiva y ampliar su rango de manejar las situaciones problemáticas. Como evidencia, una detallada revisión efectuada por Dennis y Hodnett (8), numerosos estudios han documentado la eficacia de la TCC en la DPP (42,60-64), lo que ha sido confirmado en estudios posteriores (65-67) y revisiones sucesivas (68). De esa manera, el National Institute for Health and Clinical Excellence recomienda actualmente a la TCC como tratamiento de primera línea de la DPP leve o moderada (56). Sin embargo, la tasa de deserción es bastante elevada, ya que entre el $10-40 \%$ de las participantes no termina o completa el proceso de TCC propuesto, principalmente a causa del tiempo y de los altos costos asociados (8).

Por tal razón, algunos autores han propuesto la TCC de grupo para reducir los costos y las listas de espera, pero los datos de estudios que apoyen dicha alternativa son todavía escasos y no permiten extraer conclusiones ciertas o indicar guías de tratamiento basadas en evidencia (69).

Algunos estudios han demostrado que la combinación entre TCC y $A D$ resulta más eficaz que la sola farmacoterapia en el tratamiento de la depresión grave, permitiendo obtener el máximo beneficio con los mínimos efectos negativos, pero todavía son pocos los estudios que han analizado la eficacia de la terapia combinada TCC y AD en el tratamiento de la DPP $(42,70)$.

Por todo lo anterior es que este trabajo pretende examinar a la luz de las evidencias disponibles, la eficacia de la TCC en la prevención de la DPP en mujeres consideradas de riesgos durante el embarazo. 


\section{MÉTODO}

Se ha efectuado una búsqueda de la literatura a través de datos bibliográficos electrónicos con el fin de identificar los estudios focalizados sobre la eficacia de la TCC en la prevención de la DPP, en mujeres consideradas como población de riesgo durante el embarazo.

Los límites temporales han sido considerados de enero de 1991 a junio de 2012. Las bases de datos electrónicas analizadas han sido: PubMed, Medline, Embase, Cochrane Library y Psyc INFO.

Han sido utilizadas las siguientes palabras claves: "depresión prenatal", "depresión posparto", "preparto", "posparto", "embarazo", "terapia cognitivoconductual", "prevención" y "tratamiento".

Han sido considerados los estudios en inglés. Para tener una descripción detallada de la metodología utilizada en los estudios, así como para tener datos más detallados de los mismos, han sido excluidos los documentos publicados como abstract, cartas al editor o reporte de casos.

Siguiendo los criterios antes mencionados, han sido seleccionados 24 artículos. De los cuales 9 se trataban de revisiones de la literatura y, en consecuencia, no han sido tomados en consideración. Por lo tanto, los restantes 15 estudios potencialmente relevantes han sido examinados y evaluados de acuerdo a los criterios de admisibilidad y de calidad metodológica por parte de revisores, cuyos resultados han sido confrontados, y las divergencias han sido resueltas mediante la discusión acorde a criterios previamente establecidos.

Al término de esta revisión y confrontación, sólo 8 de los 15 estudios han sido considerados idóneos para la inclusión en la revisión porque presentaban las siguientes características: protocolos randomizados y controlados, focalización en la eficacia de la TCC como estrategia para la prevención de la DPP en las mujeres en riesgo durante el embarazo.

\section{RESULTADOS}

Chabrol y cols (71), realizaron un estudio randomizado controlado (RCT) para valorar la eficacia de un programa con fines a la prevención y al tratamiento de la DPP suministrando la Escala de Depresión Posnatal de Edimburgo (Edimburgh Postnatal Depression Scale, EPDS), la cual ha sido validada en múltiples países e idiomas, incluido Chile (72). De las 859 mujeres a las que se les administró el instrumento, 258 obtuvieron un puntaje que las ubicaba en riesgo de depresión; ellas fueron asignadas al grupo de prevención/tratamiento $(n=130)$ o al grupo control $(n=128)$. El grupo de prevención recibió una sesión de TCC tras el parto. Entre la cuarta y sexta semanas tras el mismo, todas las participantes fueron reevaluadas con la EPDS. En el retest, las mujeres del grupo de prevención han mostrado una reducción significativa de los síntomas depresivos. Aquellas que tenían un puntaje de la EPDS $\geq 11$ han sido evaluadas nuevamente utilizando la Escala de Evaluación para la Depresión de Hamilton (Hamilton Depression Rating Scale, HDRS), el Inventario de Depresión de Beck (Beck Depression Inventory, BDI) y la Entrevista Neuropsiquiátrica Internacional (Mini-Neuropsychiatric Interview, MINI). En los casos en los cuales se observó un diagnóstico de episodio depresivo mayor, no obstante el programa preventivo, las mujeres participantes han proseguido en el grupo de tratamiento $(n=18)$ o en el grupo de control $(n=30)$. Las mujeres han sido tratadas con $5-8$ sesiones domiciliarias de TCC de 1 hora de duración. La intervención efectuada fue bien aceptada, reduciendo significativamente los síntomas depresivos respecto del grupo de control y ha resultado eficaz para la prevención y el tratamiento de la DPP.

Hagan y cols (73), testearon la eficacia de un programa de prevención con TCC para reducir la prevalencia de depresión durante el primer año del posparto en madres con parto prematuro. Fueron incluidas en el estudio todas las madres de niños de pretérmino ( $<33$ semanas) o con un muy bajo peso al nacer $(<1.500 \mathrm{~g})$. Durante la segunda semana después del parto, las participantes han sido evaluadas mediante el Inventario para los Trastornos Afectivos y la Esquizofrenia (Schedule for Affective Disorders and Schizophrenia, SADS). Las mujeres que resultaron encontrarse en riesgo de desarrollar la DPP fueron randomizadas en el grupo de intervención $(n=101)$ o en el grupo de control $(n=98)$. La intervención consistió en 6 sesiones semanales de TCC de grupo con una duración de 2 horas, entre la segunda y la sexta semana después del parto. Todas las participantes del estudio fueron nuevamente evaluadas con la SADS a 2, 6 y 12 meses después del parto pretérmino. Cincuenta y cuatro mujeres $(27 \%)$ fueron diagnosticadas como afectadas por depresión menor o mayor. En este estudio no se evidenció diferencias significativas entre los dos grupos en la prevalencia, en la aparición y en la duración de los episodios de depresión, sin embargo, las participantes evaluaron el programa de intervención como útil y oportuno.

Zayas y cols (74), condujeron un RCT para testear la eficacia de una intervención psicosocial a bajo costo en el reducir la DAP y la DPP. Para aquello, tomaron como muestra a mujeres de nivel socioeconómico bajo y pertenecientes a minorías étnicas $(n=187)$, consideradas en riesgo de 
depresión, asignándolas al grupo de intervención $(n=57)$ o al grupo de control $(n=43)$. La intervención consistió en 8 sesiones de TCC dirigida a la prevención de la DPP, 4 sesiones de psicoeducación y apoyo social continuo. La revaloración ha sido realizada 3 meses después del parto. El grupo de intervención no ha evidenciado diferencias significativas en el funcionamiento social o en los síntomas depresivos.

Muñoz y cols (75), evaluaron una intervención prenatal para prevenir la aparición de DAP o DPP. La TCC fue dirigida al manejo del ánimo, a las competencias sociales, como a la modalidad de apego. Cuarenta y un mujeres embarazadas y de alto riesgo de desarrollar una DPP fueron asignadas al grupo de intervención ( $n=21)$ o al grupo control $(n=20)$. La evaluación de eventuales síntomas depresivos fue obtenida durante el embarazo y a 1, 3, 6 y 12 meses tras el parto. Si bien se encontró diferencia en la incidencia de episodios depresivos mayores entre los dos grupos (14\% en el grupo de intervención vs $25 \%$ en el grupo control), no presentó significación estadística, tal vez a causa del tamaño reducido de la dimensión de la muestra.

Austin y cols (76), evaluaron la eficacia de la TCC en mujeres con DAP leve o moderada, consideradas en riesgo de DPP. Ellas fueron asignadas al grupo de tratamiento o al grupo de control al cual se ha dado un tríptico informativo sobre cómo manejar los problemas que podrían aparecer en el embarazo. La EPDS y el Inventario de Ansiedad Rasgo-Estado (State-Trait Anxiety Inventory, STAI) fueron administradas antes de comenzar la intervención (T1), apenas concluida la intervención (T2), dos meses después del parto (T3) y cuatro meses después del parto (T4). De las 277 mujeres (EPDS $>10)$ que aceptaron participar, 191 fueron asignadas al grupo TCC y 86 al grupo de control. El grupo TCC recibió 6 sesiones semanales de 2 horas de terapia, centrada en la prevención y el manejo del estrés, de la ansiedad y de la DAP, así como el cuidado del neonato. Los resultados han demostrado que en todas las mujeres se logró una mejora significativa de la sintomatología depresiva y ansiosa, y que la intervención mediante TCC no fue significativamente más eficaz respecto a las informaciones dadas al grupo de control. Hay que tener presente que los resultados pueden encontrarse afectados por la tendencia a la remisión espontánea (que se puede dar en sujetos leve o moderadamente sintomáticos) y la posibilidad que el hecho de lograr empáticamente un auxilio informativo práctico, como el recibido por los sujetos del grupo de control, haya tenido un valor terapéutico (activando mecanismos de coping).
Cho y cols (77), realizaron un RCT piloto para valorar la eficacia de la TCC en la prevención de la DPP en mujeres en riesgo. Novecientas veintisiete mujeres embarazadas fueron reclutadas de 6 centros obstétricos y puestas a screening con la BDI. De las 99 mujeres con puntajes significativamente elevados en la BDI (puntaje >16), 27 fueron diagnosticadas como deprimidas mediante la Entrevista Clínica Estructurada (SCID-I), de las cuales 15 fueron asignadas a TCC y 12 al grupo de control. La intervención consistió en 9 sesiones quincenales de 1 hora centrada en la mejora del ánimo deprimido y sobre relaciones de pareja disfuncionales. Los resultados demostraron que la TCC fue eficaz en la reducción de síntomas depresivos y mostraron, también, un efecto persistente en al menos un mes después del parto.

El-Mohandes y cols (78), evaluaron la eficacia de una intervención de TCC realizada durante el embarazo, en la reducción de los siguientes riesgos conductuales en el período posnatal: humo directo, exposición ambiental al humo de tabaco, depresión y exposición a violencia doméstica por parte de la pareja. Mujeres afroamericanas en 6 clínicas del Distrito de Columbia fueron evaluadas, reclutadas y asignadas al grupo de intervención con TCC $(n=452)$ o al grupo de control con tratamiento habitual $(n=461)$. Los datos fueron obtenidos en el período prenatal y en una media de 10 semanas después del parto. Fueron realizadas 2 sesiones en el posparto, encontrándose una reducción importante en lo que respecta a la presencia de la depresión, incluso si la diferencia entre los dos grupos no ha resultado estadísticamente significativa.

Le y cols (79), realizaron un RCT para evaluar la eficacia de una intervención con TCC en la prevención de la DPP en mujeres de alto riesgo durante el embarazo. Una muestra de 217 participantes, prevalentemente inmigrantes de Centro América de bajos recursos, fueron asignadas al grupo de intervención $(n=112)$ con 8 semanas de TCC de grupo durante el embarazo y 3 sesiones de tratamiento individual en el post-parto, o al grupo de control $(n=105)$. Las participantes fueron evaluadas en 5 momentos sucesivos con la escala de depresión del Centro para Estudios Epidemiológicos (Center for Epidemiological Studies Depression Scale, CES-D), la BDI y el Mood Screener (MS) con el fin de identificar eventuales síntomas depresivos o la aparición de episodios depresivos mayores. El grupo de intervención presentó menor sintomatología depresiva y significativamente menor incidencia de episodios depresivos respecto al grupo de control al segundo momento. Tales efectos han resultado más marcados en las mujeres que habían participado enteramente en las 
sesiones de intervención. Más aún, en las evaluaciones sucesivas la incidencia acumulativa de episodios depresivos mayores no reportó diferencias estadísticamente significativas entre los dos grupos. Tal intervención ha resultado eficaz para reducir la DAP, pero no la DPP.

\section{DISCUSIÓN}

Si bien el rol de la TCC en el tratamiento de la depresión mayor $(59,80)$ y de la DPP $(56,62,63)$ ha sido bien documentado, sólo pocos estudios se han ocupado de su rol en la prevención de la DPP en mujeres identificadas en riesgo durante el embarazo, como había ya puesto en evidencia la investigación de Morrel y cols (68).

Los estudios efectuados con este método muestran todavía algunas limitaciones metodológicas. En primer lugar, resultan heterogéneas tanto en las metodologías utilizadas para evaluar el riesgo de desarrollar un estado depresivo en una mujer embarazada como en las medidas de éxito. Por tal motivo es difícil realizar una comparación directa entre los resultados obtenidos en los estudios examinados. Por ejemplo, en algunos no ha sido utilizada una metodología adecuada para la evaluación diagnóstica de la DPP: sólo en los estudios de Hagan y cols (73) y de Cho y cols (77) han sido utilizadas entrevistas estructuradas estandarizadas (la SCID-I y la SADS). Además, en algunos estudios, la muestra es muy limitada para permitir un análisis estadístico confiable entre los grupos y, en algunos casos, han sido estudiadas categorías muy específicas para permitir una adecuada generalización de los resultados (por ejemplo: mujeres de NSE bajo, afroamericanas o madres de prematuros).

Otra importante limitación ha sido la falta de un adecuado seguimiento en el posparto: sólo 3 estudios han considerado un período de control de 12 meses $(73,75,79)$. Como se sabe, la DPP puede no desarrollarse en seguida (por ejemplo, tras un año del parto), por lo que un período más largo de observación es fundamental para definir mejor la eficacia de una estrategia de prevención a largo plazo.

Como se ha observado, las intervenciones de TCC adoptadas en los estudios han sido efectuadas utilizando sólo pocas sesiones, por lo que estas estrategias de intervención deben ser consideradas más como "psicoterapia breve", que una verdadera TCC. Se han centrado en los procesos de coping asociados a situaciones de estrés, más que a formas de psicoterapia estructurada y a "largo plazo". Estos aspectos pueden explicar porqué todos los enfoques de TCC utilizados en estos estudios han producido un mejoramiento en el corto plazo, mientras falta definir su real eficacia en la depresión en el largo plazo. Convencer a las mujeres embarazadas en riesgo de depresión, pero sin una sintomatología significativa, a participar de un programa preventivo resulta complejo y está asociado a elevadas tasas de deserción (75).

En conclusión, sólo dos de los estudios seleccionados han demostrado una eficacia estadísticamente significativa de la TCC versus otras estrategias usadas en los grupos de control, por lo que son necesarios estudios controlados posteriores a largo plazo para establecer con datos certeros, el impacto real de este enfoque psicoterapéutico en la prevención de la DPP.

La presente revisión permite evidenciar algunas importantes implicaciones clínicas y psicoterapéuticas. Primero que todo, el diagnóstico precoz y el tratamiento de la DAP revisten de una importancia significativa para evitarle a la mujer con depresión no tratada la aparición de la DPP. Por lo mismo, los síntomas de malestar referidos por las mujeres embarazadas no deben ser subvalorados ni considerados como un componente normal de la experiencia del embarazo. Prestar mayor atención a los síntomas de la depresión, puede en efecto mejorar el screening y la identificación de mujeres que sufren de depresión durante el embarazo y el posparto (5). Además, para las mujeres que están viviendo un episodio depresivo inicial leve o moderado durante el embarazo, la TCC representa el tratamiento de primera elección, considerando el hecho de que la mayor parte de las mujeres embarazadas afectadas por síntomas depresivos rechaza consumir fármacos durante el embarazo. Si bien al momento sólo hay disponibles pocos estudios sobre la eficacia de la TCC en la DAP (81), no evidencian una diferencia en la respuesta entre las mujeres embarazadas deprimidas y la población general.

Por otra parte, si la depresión es grave o no responde a la psicoterapia sola, se recomienda una combinación entre fármacos y TCC $(48,56,57)$. Al respecto, una reciente revisión de De Rubeis y cols (59) con imagen funcional de resonancia magnética (fMRI) demuestra que la TCC resulta tener una eficacia similar a aquella de la mayor parte de los fármacos antidepresivos, reduciendo el riesgo de recaída, incluso después de una eventual interrupción de la terapia farmacológica.

La TCC probablemente actúa sobre los mismos mecanismos neuronales involucrados en la terapia con $A D$, no a través de un proceso "bottom-up" sino que mediante un proceso "top-down" (82). La misma revisión sugiere que los pacientes con mayor reactividad límbica serían los principales 
candidatos para una fármacoterapia, mientras que aquellos con dificultad en la regulación de las emociones, especialmente si se asocian a una disminución de la funcionalidad prefrontal, podrían beneficiarse principalmente de la TCC.

Entre los enfoques de TCC disponibles, el terapeuta debiese adoptar la estrategia más adecuada, teniendo en cuenta las características de la personalidad, las capacidades cognitivas, la motivación, la gravedad de la depresión y la eventual presencia de una comorbilidad psiquiátrica. Asimismo, debe ser evaluada la duración de la terapia y la frecuencia de las sesiones, que deben ser individualizadas para cada paciente.

En particular, el nuevo modelo cognitivo denominado como "post-racionalista" (83) puede ser sugerido en las mujeres que sientan una necesidad de un cambio emotivo profundo, de manera de incidir en sus procesos de apego, con el fin de desarrollar una relación más adaptativa con el niño (84). Los principales temas desarrollados por el postracionalismo son los siguientes:

- El desarrollo del sí está estrechamente ligado a las activaciones afectivas y emotivas $(85,86)$.

- En la interface existente entre la experiencia inmediata y la explicación, como entre la modalidad subjetiva de ordenar y autorreferirse la experiencia, se construye activamente el sentido de sí y de los otros (87).

- La psicopatología es recomendada cuando la asimilación de una nueva experiencia aparece como perturbante y no puede ser asimilada en el sentido de sí sin provocar una alteración de la coherencia interna (88).

- La descompensación que se produce puede ser resuelta sólo a través del logro de un nuevo equilibrio más complejo y adaptativo (89).

- Existe un continuum entre normalidad y patología: así la severidad de los síntomas depende de un compromiso en la capacidad de flexibilidad, de generatividad, de autorregulación y de abstracción (90-93).

En mujeres con un episodio depresivo o consideradas con riesgo de DPP, utilizando el modelo post-racionalista, los sentimientos de negatividad percibidos como aspectos de sí objetivos e inmutables, pueden ser focalizados como expresión de la manera propia de organizar la experiencia subjetiva, mejorando así los recursos adaptativos personales. En todo caso, la elección de la TCC sola o en asociación con los fármacos $\mathrm{AD}$, debe ser evaluada caso a caso y requiere decisiones tomadas en colaboración entre médico y paciente.

\section{CONCLUSIÓN}

Como ha demostrado esta revisión, se han efectuado sólo pocos estudios controlados sobre la eficacia de la TCC en la prevención de la DPP en las mujeres en riesgo en el curso del embarazo. Es más, los estudios hasta ahora publicados presentan algunos límites metodológicos relevantes. Así, resulta importante que sean efectuadas investigaciones utilizando una metodología y un seguimiento más adecuados, para evaluar mejor el rol de la TCC en la prevención de la DPP. En todo caso, entre las principales intervenciones psicoterapéuticas disponibles, la TCC es considerada una estrategia de primera línea en el tratamiento de la depresión mayor. Resulta así importante evaluar en estas mujeres la eficacia de los nuevos modelos cognitivos como el post-racionalista en cuanto están potencialmente en condición de producir un más eficaz y estable reordenamiento emocional.

\section{REFERENCIAS}

1. Bennett HA, Einarson A, Taddio A, Koren G, Einarson TR. Prevalence of depression during pregnancy: systematic overview. Obstet Gynecol 2004;103:698-709.

2. Gavin NI, Gaynes BN, Lohr KN, Meltzer-Brody S, Gartlehner G, Swinson T. Perinatal depression. A systematic review of prevalence and incidence. Obstet Gynecol 2005;106:1071-83.

3. Gaynes BN, Gavin N, Meltzer-Brody S, et al. Perinatal depression: prevalence, screening accuracy, and screening outcomes. Evid RepTechnol Assess 2005;119:1-8.

4. Banti S, Mauri M, Oppo A, et al. From the third month of pregnancy to 1 year postpartum. Prevalence, incidence, recurrence, and new onset of depression. Results from the Perinatal Depression-Research \& Screening Unit study. Compr Psychiatry 2011;52:343-51.

5. Manber R, Blasey C, Allen JJB. Depression symptoms during pregnancy. Arch Women Ment Health 2008;11:43-8.

6. Raudzus J, Misri S. Managing unipolar depression in pregnancy. Curr Opin Psychiatry 2008;22:13-8.

7. Gotlib IH, Whiffen VE, Mount JH, Milne K, Cordy NI. Prevalence rates and demographic characteristics associated with depression in pregnancy and the postpartum. J Consult Clin Psychology 1989;57:269-74.

8. Dennis CL, Hodnett E. Psychosocial and psychological interventions for treating postpartum depression (Review). Cochrane Database of Systematic Reviews 2008; Issue 3.

9. Dennis CL. Preventing postpartum depression Part II: a critical review of nonbiological interventions. Can $\mathrm{J}$ Psychiatry 2004;49:526-38.

10. Dennis CL. Psychosocial and psychological interventions for prevention of postnatal depression: systematic review. BMJ 2005;331:15. 
11. Ryan D, Milis L, Misri N. Depression during pregnancy. Can Fam Physician 2005;51:1087-93.

12. Cox J, Murray D, ChapmanG. A controlled study of the onset, duration and prevalence of Postnatal Depression. Br J Psychiatry1993;163;27-31.

13. Gjerdingen D.The effectiveness of various postpartum depression treatments and the impact of antidepressant drugs on nursing infants. J Am Board Fam Pract 2003;16:372-82.

14. O'Hara M, Swain A. Rates and risk of postpartum depression - a meta-analysis. International Review Psychiatry 1996;8:37-54.

15. O'Hara M, Zekoski E. Postpartum depression: a comprehensive review. Motherhood and Mental IIIness1988;2:17-63.

16. BrownS, Lumley J. Satisfaction with care in labour and birth: A survey of 790 australian women. Birth 1994;21:4-13.

17. Jadresic E. Depresión postparto. Rev Chil Neuro-Psiquiat 1990;28:147-58.

18. Patel V, De Souza N, Rodrígues M. Postnatal depression and infant growth and development in low income countries: a cohort study from Goa, India. Arch Dis Child 2002;87:1-4.

19. Cooper P, Murray L. Postnatal depression. BMJ 1998;316:1884-6.

20. Cooper PJ, Tomlinson M, Swartz L, Woolgar M, Murray $\mathrm{L}$, Molteno $\mathrm{C}$. Postpartum depression and the mother-infant relationship in a South African peri-urban settle $\neg$ ment. Br J Psychiatry 1999;175:554-8.

21. Jadresic E, Jara C, Miranda M, Arrau B, Araya R. Trastor $\neg$ nos emocionales en el embarazo y el puerperio: estudio prospectivo de 108 mujeres. Rev Chil Neuro-Psiquiat 1992;30:99-106.

22. Alvarado R, Rojas M, Monardes J, Neves E, Olea E, Perucca E, et al. Cuadros depresivos en el postparto y variables asociadas en una cohorte de 125 mujeres em $\neg$ barazadas. Rev Psiquiat 1992;3:1168-76.

23. MINISTERIO DE SALUD. Guía Clínica: tratamiento de personas con depresión, 2009. Hallado en: http:// www.minsal.gob.cl/portal/url/item/7222754637c08646 e04001011f014e64.pdf

24. American Psychiatric Association. Diagnostic and Statistical Manual of Mental Disorders, Forth Edition (DSM-IV). Washington: APA Press, 1994.

25. Pitt B. "Atypical" depression following childbirth. BrJ Psychiatry 1968;114:1325-35.

26. Kennerly H, Gath D. Maternity blues: detection and measurement by questionnaire. $\mathrm{Br} \mathrm{J}$ Psychiatry 1989;155:356-62.

27. O'Hara M, SchlechteJ, Lewis D. Prospective study of postpartum blues: biologic and psycosocial factors. Arch General Psychiatry 1991;48: 801-5.

28. Gregoire A. Hormones and postnatal depression. Br J Midwifery 1995;3:99-105.

29. OakleyA, Chamberlaing. Medical and social factors in post-partum depression. J Obstet Gynaecol 1981;1:182-7.

30. Hipwell A, Kumar R. Le conseguenze della psicosi post-partum di tipo affettivo sul bambino. En: Murray,
L. y Cooper, P. (Ed). Depressione del post-partum e sviluppo del bambino, CIC Edizioni Internazionali, Roma. 1997.

31. Brockington I, Cernik K, Schofield E, Downing A, Francis A, Keelan C. Puerperal psychosis: phenomena and diagnosis. Arch General Psychiatry 1981;38:829-33.

32. Bramante A. 2005.Fare e disfare... dall'amore alla distruttivita. II figlicidio materno, Aracne, Roma.

33. Menacci C, Anniverso R. (Ed.) 2005. Le manifestazioni psichiche nei cicli vitali della donna, Pacini, Pisa.

34. Zaccagnino M. 2009.I disagidella maternita. Individuazione, prevenzione, trattamento. Franco Angeli, Milano.

35. Bonari L, Pinto N, Ahn E, Einarson A, Steiner M, Koren $\mathrm{G}$. Perinatal risks of untreated depression during pregnancy. Can J Psychiatry 2004;49:726-35.

36. Misri S, Kendrick BA, Oberlander TF, et al. Antenatal depression and anxiety affect postpartum parenting stress: a longitudinal,prospective study. Can J Psychiatry 2010;55:222-7.

37. Cohen LS, Altshuler LL, Harlow BL, et al. Relapse of major depression during pregnancy in women who maintain or discontinue antidepressant treatment. JAMA 2006;295:499-507.

38. Schneid-Kofman N, Sheiner E, Levy A. Psychiatric illness and adverse pregnancy outcome. Int J Gyneacol Obstet 2008;101:53-6.

39. Pawlby S, Hay DF, Sharp D, Waters CS, O'Keane V. Antenatal depression in adolescent offspring: prospective longitudinal community-based study. J Affect Disord 2009;113:236-43.

40. Wan MW, Green J. The impact of maternal psychopathology on child-mother attachment. Arch Women Ment Health 2009;12:123-34.

41. Leahy-Warren P, McCarthy G. Postnatal depression: prevalence, mother's perspectives and treatment. $\mathrm{Br} \mathrm{J}$ Psychiatry2007;171:550-5.

42. Misri S, Reebye P, Corral M, Milis L. The use of paroxetine and cognitive-behavioral therapy in postpartum depression and anxiety: a randomized controlled trial. Can J Psychiatry 2004;65:1236-41.

43. Bonari L, Koren G, Einarson TR, Jasper JD, Taddio A, Einarson A. Use of antidepressant by pregnant women: evaluation of perception of risk, efficacy of evidence based counselling and determinants of decision making. Arch Women Ment Health 2005;8:214-20.

44. Dennis CL, Ross LE, Grigoriadis S. Psychosocial and psychological interventions for treating antenatal depression. Cochrane Database of Systematic Reviews 2007; Issue 3.

45. American College of Obstetricians and Gynecologists. ACOG Practice Bulletin Number 92.Obstet Gynecol 2008;4:1001-20.

46. Milgrom J, Martin P,Negri L. 2003. Icerca, prevenzione e strategie di intervento psicologico, Edizioni Erickson, Trento.

47. Monti F, Agostini F. 2006.La depression postnatale, Carocci Editore, Roma.

48. Bellantuono C, Migliarese G, Gentile S. Serotonin reuptake inhibitors in pregnancy and the risk of ma- 
jor malformations: a systematic review. Human Psychopharmacology 2007;22:121-8.

49. Misri S, Kendrick BA. Treatment of perinatal mood and anxiety disorders: a review. Can J Psychiatry 2007;52:489-98.

50. Hoffbrand S, Howard L, Crawley H. Antidepressant drug treatment for postnatal depression. Cochrane Database of Systematic Reviews 2001; Issue 2.

51. Dennis CL, Stewart DE. Treatment of postpartum depression, part 1: a critical review of biological interventions. J Clin Psychiatry 2004;65:1242-51.

52. Bellantuono C, Migliarese G, Maggioni F, Imperadore G. L'impiego dei farmaci antidepressivi nel puerperio. Recenti Prog Med 2007;98:29-42.

53. Dennis CL, Chung-Lee L. Postpartum depression helpseeking barriers and maternal treatment preferences: a qualitative systematic review. Birth 2006;33:323-31.

54. American Academy of Pediatrics Committee on Drugs. Transfer of drugs and other chemicals into human milk. Pediatrics 2001;108:776-89.

55. Dennis CL. Treatment of postpartum depression, part 2: a critical review of non biological interventions. $J$ Clin Psychiatry 2004;65:1252-65.

56. NICE, National Institute for Health and Clinical Excellence. Antenatal and Postnatal Mental Health. Clinical Management and Service Guidance NHS: London, 2007.

57. Yonkers KA, Wisner KL, Stewart DE, et al. The management of depression during pregnancy: a report from the American Psychiatric Association and the American College of Obstetricians and Gynecologists. Gen Hosp Psychiatry 2009;31:403-13.

58. Rupke SJ, Blecke D, Renfrow M. Cognitive Therapy for Depression. Am Fam Physician 2006;73:83-6.

59. De Rubeis RJ, Siegle GJ, Hollon SD. Cognitive therapy versus medication for depression: treatment outcomes and neural mechanisms. Nature 2008;9:788-96.

60. Bennet P, Honey KL, Morgan M. A brief psycho-educational group intervention for postnatal depression. Br J ClinPsychol 2002;41:405-9.

61. Prendergast J, Austin MP. Early childhood nurse-delivered cognitive behavioural counseling for post-natal depression. Australas Psychiatry 2001;9:209-16.

62. Cooper PJ, Murray L, Wilson A, Romaniuk H. Controlled trial of the short and long-term effect of psychological treatment of post-partum depression. I. Impact on maternal mood. Br J Psychiatry 2003;182:412-9.

63. Murray L, Cooper PJ, Wilson A, Romaniuk H. Controlled trial of the short- and long-term effect of psychological treatment of post-partum depression. II. Impact on the mother-child relationship and child outcome. $\mathrm{Br}$ J Psychiatry 2003;182:420-7.

64. Morrel J. Psychological interventions for postnatal depression. Randomized controlled trial and economic evaluation. National Research Register 2004.

65. Kuosmanen L, Vuorilehto M, Kumpuniemi S, Melartin T. Postnatal depression screening and treatment in maternity and child health clinics. J Psychiatr Ment Health Nurs 2010;17:554-7.

66. Slade P, Morrell CJ, Rigby A, Ricci K, Spittlehouse J, Brugha TS. Postnatal women's experiences of mana- gement of depressive symptoms: a qualitative study. Br J Gen Pract 2010;60:440-8.

67. Milgrom J, Holt CJ, Gemmill AW, Ericksen J, Leigh B, Buist A, Schembri C. Treating postnatal depressive symptoms in primary care: a randomized controlled trial of GP management, with and without adjunctive counseling. BMC Psychiatry 2011;11:95.

68. Morrel CJ, Warner R, Slade P, et al. Psychological interventions for postnatal depression: cluster randomized trial and economic evaluation. The PoNDER trial. Health Technol Assess 2009;13:1-146.

69. Stevenson MD, Scope A, Sutcliffe PA, Booth A, Slade $P$, Parry G, Saxon D, Kalthenthaler E; group cognitive behavioural therapy for postnatal depression advisory group. Group cognitive behavioural therapy for postnatal depression: a systematic review of clinical effectiveness, cost-effectiveness and value of information analyses. Health Technol Assess 2010;14:1-107.

70. Appleby L, Warner R, Whitton A, Faragher B. A controlled study of fluoxetine and cognitive behavioural counselling in the treatment of postnatal depression. BMJ 1997;314:932-6.

71. Chabrol H, Teissedre F, Saint-Jean M, Teisseyre N, Roge B, Mullet E. Prevention and treatment of postpartum depression: a controlled randomized study on women at risk. Psychol Med 2002;32:1039-47.

72. Jadresic E, Araya R, Jara C. Validation of the Edin $\neg$ burgh Postnatal Depression Scales (EPDS) in Chilean postpartum women. J Psychosom Obstet Ginaecol 1995;16:187-91.

73. Hagan R, Evans SF, Pope S. Preventing postnatal depression in mothers of very preterm infants: a randomized controlled trial. BJOG 2004;111:641-7.

74. Zayas LH, McKee MD, Jankowski KRB. Adapting psychosocial intervention research to urban primary care environments: a case example. Ann Fam Med 2004;2:504-8.

75. Muñoz RF, Le HN, Ghoshlppen C, et al. Prevention of postpartum depression in low-income women: development of the mamás y bebés/mothers and babies course. Cogn Behav Pract 2007;14:70-83.

76. Austin MP, Frilingos M, Lumley J, et al. Brief antenatal cognitive behaviour therapy group intervention for the prevention of postnatal depression and anxiety: a randomized controlled trial. J Affect Disord 2008;105:3544.

77. Cho HJ, Kwon JH, Lee JJ. Antenatal cognitive-behavioral therapy for prevention of postpartum depression: a pilot study. Yonsei Med J 2008;49:553-62.

78. El-Mohandes AA, Kiely M, Joseph JG, et al. An intervention to improve postpartum outcomes in AfricanAmerican mothers: a randomized controlled trial. Obstet Gynecol 2008;112:611-20.

79. Le HN, Perry DF, Stuart EA. Randomized controlled trial of a preventive intervention for perinatal depression in high-risk Latinas. J Consult Clin Psychol 2011;79:135-41.

80. Muñoz RF, Cuijpers $P$, Smit F, Barrera AZ, Leykin, Y. Prevention of major depression. Ann Rev Clin Psychol 2010;27:181-212.

81. Steinberg SI, Bellavance F. Characteristics and 
treatment of women with antenatal and postpartum depression. Int J Psychiatry Med 1999;29:209-33.

82. Bellantuono C, Nardi B, Mircoli G, Santone G. Manuale essenziale di psichiatria. Roma: II Pensiero Scientifico Editore, 2009.

83. Moltedo A. La evolución de la obra y el modelo de Vittorio Guidano: notas histórico-biográficas. Rev Psicología 2008;17:65-85.

84. Guidano VF. The Self in Progress. New York: The Guilford Press, 1991.

85. Nardi B, Moltedo A. Rol de la relación de reciprocidad en el desarrollo de las diversas organizaciones de significado personal. Gaceta de Psiquiatría Universitaria (Chile) 2008;4:345-58.

86. NardiB, Moltedo A. Desarrollo del modelo de las organizaciones de significado personal de Vittorio Guidano: los procesos de construcción del sí en el modelo cognitivo post-racionalista evolutivo. Psicoperspectivas 2009 (Chile);8:93-111.

87. Moltedo A. Procesos de mantención de la identidad: entre la continuidad y el cambio. Psicoperspectivas 2006 (Chile);5:35-47.
88. Moltedo A,Nardi B. Processi di mantenimento dell'identità: tra continuità e cambiamento. Quaderni di Psicoterapia Cognitiva 2010;15:98-113.

89. Moltedo A. Más allá de la nosografía: la visión cognitivo procesal sistémica del síntoma. Psicoperspectivas 2004 (Chile);3:5-93.

90. Nardi B. Costruirsi. Sviluppo e adattamento del Sé neIla normalità e nella patologia. Milano: Franco Angeli, 2007.

91. Nardi B. Guidelines on the construction of a post-rationalist therapeutic approach. European Psychotherapy 2010;9:57-67.

92. Nardi B, Bellantuono C. A new adaptive and evolutionary conceptualization of the personal meaning organization (POM) framework. European Psychotherapy 2008;8:5-16.

93. Moltedo A. Le Difficoltà della Nosografia del Disturbi dell'Umore. En: B. Nardi (Ed). Disturbi dell'Umore. Ancona: Ed. Accademia Dei Cognitivi Della Marca. Italia. 2007. 